\title{
SYMMETRY EXPLOITATION IN THE NATURAL VIBRATIONS OF ROD SYSTEMS
}
A.M. Pavlov
pavlov_arseniy@mail.ru
A.N. Temnov
antt45@mail.ru

\section{Bauman Moscow State Technical University, Moscow, Russian Federation}

\section{Abstract}

The purpose of this work was to study spectral and Cauchy problem for the mechanical system consisting of three rods, two of them being identical and connected with the third one by linear elastic elements. We stated the corresponding spectral problem and studied its spectrum. Findings of the research show that eigenfunctions of the considered spectral problem are classified according to the irreducible representations of the finite group of transformations despite the fact that the initial equations system admits continuous (Lie) transformation groups. We considered the weak solution of Cauchy problem and revealed its simplification in case of special "symmetrical" form of initial conditions and right-hand side of the corresponding operator equation system

\section{Keywords}

Rod system, symmetry group, irreducible representation, spectral problem, eigenfunction classification, projection operator

Received 26.12.2016

(C) BMSTU, 2017

Introduction. In the natural vibrations problem symmetry of mechanical system plays an important role. The representation theory of symmetry groups is an approach which allows recognizing and exploiting the influence of the system symmetry on the corresponding spectral problem. The main result of the representation theory with respect to spectral problems can be formulated in the following theorem [1].

Theorem. Let linear operator A commutes (permutable) with the representation operators of symmetry group $G$ and has a discrete spectrum of eigenvalues with finite multiplicity, then its eigenfunctions are basis functions of group $G$ irreducible representations.

As a result, with help of projection operators on subspaces of irreducible representations, spectral problem can be solved in these subspaces [2] which usually have lower dimension than initial space [3].

This approach has been successfully applied to mechanical systems with a finite number of degrees of freedom [4] (molecular vibrations [5], mass-spring models of mechanical systems [6, 7], finite element and finite difference models [8-10] etc.). In this case the symmetry group is a finite group, usually represented by spatial symmetry of the system, and the irreducible representations and their respective projectors can be easily found with the tables of characters of the finite groups irreducible representations [11]. 
If the mechanical system includes a continuous medium, it is much more difficult to determine the symmetry group only out of the spatial symmetry, since the displacements of the system are described by differential equations in partial derivatives, which generally have the continuous symmetry groups [12].

The complete set of differential equation system symmetry groups can be obtained by universal algorithms [13]. And it is also possible to write irreducible representations and corresponding projectors for continuous groups [14]. But the irreducible representations of continuous groups were found only for some particular cases [15]. For an arbitrary continuous group, finding them is quite a challenge. Also for the boundary value problems, this approach requires the invariance of the manifold defined by boundary conditions, which is not always possible to follow [16].

But there are special cases when obtained continuous symmetry groups illustrate linearity of the operator, corresponding to the original differential equations system, and in addition includes some finite group of permutations. Then, taking into account that eigenfunctions set is not invariant under linear transformations, eigenfunctions of this operator can be classified according by the irreducible representations of obtained permutations group or its subgroup, with respect to which the boundary conditions are invariant.

In this paper one of such cases was considered. Using an approach based on the theory of both finite and continuous groups we have obtained and then verified symmetry classification of eigenfunctions of the operator, generated by the natural vibrations problem of the rod system.

The problem statement. We will consider a mechanical system consisting of three linear elastic rods of length $l$, two of which have the same inertia and stiffness characteristics and are connected by linear-elastic connections with a third rod different from the first two, in crosssection with the coordinate $x=l$. We denote the stiffness and distributed mass of the rods as $p_{j}(x)$ and $m_{j}(x), j=0,1,2$ respectively for central and lateral rods. Functions $p_{j}(x) \in \mathrm{C}^{1}[0, l], \quad m_{j}(x) \in \mathrm{C}^{0}[0, l], \quad j=0,1,2$ are bounded positive functions of coordinate $x$.

Displacements of the rods cross-sections

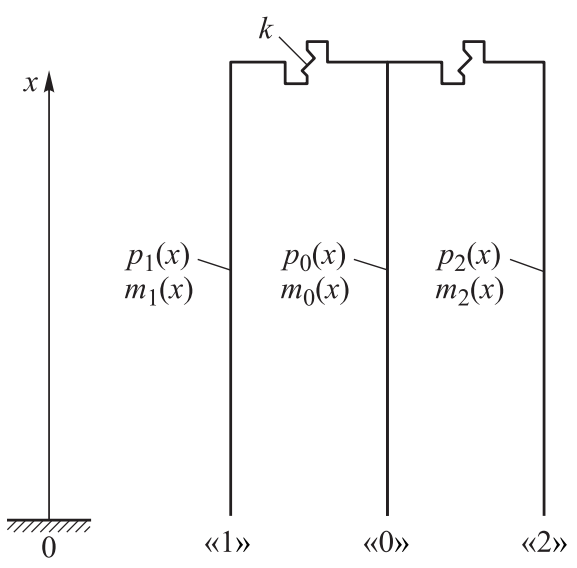

Fig. 1. Rod system of three rods $u_{j}(x, t), j=0,1,2$ along the axis $X$ are defined by the equations

$$
m_{0}(x) \frac{\partial^{2} u_{0}(x, t)}{\partial t^{2}}-\frac{\partial}{\partial x}\left(p_{0}(x) \frac{\partial u_{0}(x, t)}{\partial x}\right)=q_{0}(x, t) ;
$$




$$
\begin{aligned}
& m_{1}(x) \frac{\partial^{2} u_{1}(x, t)}{\partial t^{2}}-\frac{\partial}{\partial x}\left(p_{1}(x) \frac{\partial u_{1}(x, t)}{\partial x}\right)=q_{1}(x, t) \\
& m_{2}(x) \frac{\partial^{2} u_{2}(x, t)}{\partial t^{2}}-\frac{\partial}{\partial x}\left(p_{2}(x) \frac{\partial u_{2}(x, t)}{\partial x}\right)=q_{2}(x, t) .
\end{aligned}
$$

Where $q_{j}(x), j=0,1,2$ are bounded functions of coordinate $x$ which determine the external loads.

Boundary conditions for cross sections with coordinates $x=0$ and $x=l$ are given by

$$
\begin{aligned}
& u_{0}^{\prime}(0, t)=u_{1}^{\prime}(0, t)=u_{2}^{\prime}(0, t)=0 ; \\
& p_{1}(l) u_{1}^{\prime}(l, t)+k\left(u_{1}(l, t)-u_{0}(l, t)\right)=0 ; \\
& p_{2}(l) u_{2}^{\prime}(l, t)+k\left(u_{2}(l, t)-u_{0}(l, t)\right)=0 ; \\
& p_{0}(l) u_{0}^{\prime}(l, t)+k\left(2 u_{0}(l, t)-u_{1}(l, t)-u_{2}(l, t)\right)=0 .
\end{aligned}
$$

To state evolutionary problem we will add to (1), (2) the initial conditions

$$
\begin{aligned}
& u_{j}(x, 0)=\psi_{j}^{0}(x) ; \\
& \frac{\partial u_{j}}{\partial t}(x, 0)=\psi_{j}^{1}(x), j=0,1,2 .
\end{aligned}
$$

Operator statement. We assume Hilbert space

$$
H=\left(\sum_{j=0}^{2} \oplus L_{2 j}\left([0, l], m_{j}(x)\right)\right) \Theta\{1\}^{\mathrm{T}},
$$

vector functions $U(t)=\left\{u_{j}(t)\right\}^{\mathrm{T}}$, where $u_{j}(t) \in C^{2,2}\left(L_{2 j}\left([0, l], m_{j}(x)\right),[0, \infty)\right)$. Vector $F(t)=\left\{f_{j}(t)\right\}^{\mathrm{T}}$, where $f_{j}(t) \in C\left([0, \infty), L_{2 j}\left([0, l], m_{j}(x)\right)\right)$ and $f(x, t)=$ $=\frac{q_{j}(x, t)}{m_{j}(x)}$.

The inner product in $H$ is defined by

$$
(\mathrm{V}, \mathrm{W})=\sum_{j=0}^{2} \int_{0}^{l} m_{j}(x) v_{j} w_{j} d x
$$

Let the matrix operator $A=\operatorname{diag}\left(-\frac{1}{m_{j}(x)} \frac{\partial}{\partial x}\left(p_{j}(x) \frac{\partial}{\partial x}\right)\right)$ has domain $D(A): \Omega U(t)=0$, where $\Omega$ is an operator of the boundary conditions (2).

From (1)-(3), we obtain the nonhomogenious Cauchy problem for the hyperbolic operator equation: 


$$
\begin{gathered}
\ddot{U}(t)+A U(t)=F(t) ; \\
U(0)=\Psi^{0}, \dot{U}(0)=\Psi^{1} .
\end{gathered}
$$

Spectral problem for the operator $A$. Let us assume $F(t)=0, U(t)=U e^{i \omega t}$, where $U$ is the eigenfunction, $\omega$ is the natural frequency of the rod system. We obtain the spectral problem for the operator $A$

$$
A U-\lambda U=0,
$$

where $\lambda=\omega^{2}$.

Lemma 1. The operator $A$ is unbounded, self-adjoint and uniformly positive in $H$ operator. The squared norm in the energetic space of the operator $A$ is given by

$$
\|U\|_{A}^{2}=\sum_{j=0}^{2} \int_{0}^{l} p_{j}(x)\left(\frac{\partial u_{j}}{\partial x}\right)^{2} d x+k \sum_{j=1}^{2}\left(u_{0}(l)-u_{j}(l)\right)^{2}=2 \Pi>0 .
$$

Proof. Uboundedness of operator $A$ directly follows from that every matrix element of $A$ is ubounded. Direct evaluation of $(A U, V),(U, A V)$ and $(A U, U)$ shows that $A$ is self-adjoint and uniformly positive in $H$.

Similarly to work [17], we formulate a theorem on the spectrum of the operator $A$.

Theorem 1. The operator $A$ has a positive real discrete spectrum of eigenvalues

$$
0<\lambda_{1}<\lambda_{2}<\ldots<\lambda_{k}<\ldots, \quad \lambda_{k} \rightarrow \infty
$$

and the system of complete and orthogonal in spaces $H$ and $H_{A}$ eigenfunctions $\left\{U_{k}\right\}_{k=1}^{\infty}$, with the following orthogonality conditions

$$
\begin{gathered}
\left(U_{k}, U_{s}\right)=\sum_{j=0}^{2} \int_{0}^{l} m_{j}(x) u_{k j} u_{s j} d x=\delta_{k s} ; \\
\left(U_{k}, U_{s}\right)_{A}=\sum_{j=0}^{2} \int_{0}^{l} p_{j}(x)\left(\frac{\partial u_{k j}}{\partial x}\right)\left(\frac{\partial u_{s j}}{\partial x}\right) d x+ \\
+k \sum_{j=1}^{2}\left(u_{0}(l)-u_{k j}(l)\right)\left(u_{0}(l)-u_{s j}(l)\right)=\lambda \delta_{k s} .
\end{gathered}
$$

Proof. Positive and real properties of the spectrum follow from Lemma 1. Discreteness of spectrum follows from Sobolev's embedding theorem which is proved for this problem by comparison of norms in space $H_{A}$ and Sobolev space $W_{2}^{1}$.

Lie symmetries analysis of the spectral problem. We will consider that inertialmass characteristics of the rods do not depend on the coordinate $x$

$$
\begin{aligned}
& p_{1}(x)=p_{2}(x)=p_{s} ; \\
& p_{0}(x)=p_{0} ; \\
& m_{1}(x)=m_{2}(x)=m_{s} ; \\
& m_{0}(x)=m_{0} .
\end{aligned}
$$


Problem (6) corresponds to a system of differential equations

$$
\begin{aligned}
& \frac{p_{0}}{m_{0}} \frac{d^{2} u_{0}(x)}{d x^{2}}+\lambda u_{0}(x)=0 ; \\
& \frac{p_{s}}{m_{s}} \frac{d^{2} u_{1}(x)}{d x^{2}}+\lambda u_{1}(x)=0 ; \\
& \frac{p_{s}}{m_{s}} \frac{d^{2} u_{2}(x)}{d x^{2}}+\lambda u_{2}(x)=0 .
\end{aligned}
$$

Let the $M \subset X \times U$ is open subset of space $X \times U$, where $X:=\{x\}$, $U:=\left\{u_{0}, u_{1}, u_{2}\right\}^{\mathrm{T}}$. We define the space $X \times U \times U^{(1)} \times U^{(2)}$, which is a second prolongation of space $X \times U$. Here $U^{(i)}:=\left\{\frac{d^{i} u_{0}}{d x^{i}}, \frac{d^{i} u_{1}}{d x^{i}}, \frac{d^{i} u_{2}}{d x^{i}}\right\}^{\mathrm{T}}, i=1,2$. In the space $X \times U \times U^{(1)} \times U^{(2)}$ the subset $M^{(2)}$ corresponds to a subset $M$.

Mapping $\Delta=\left\{\Delta_{1}, \Delta_{2}, \Delta_{3}\right\}$ defined by left-hand side of system (9) translates $M^{(2)}$ into three-dimensional Euclidean space: $\Delta: M^{(2)} \rightarrow \mathbb{R}^{3}$. System (9) defines a subvariety $\wp_{\Delta}^{(2)}=\left\{\mu \in M^{(2)}: \Delta(\mu)=0\right\}$ consisting of its solutions.

Definition 1 [13]. A local group of transformations $G$ acting on an open subset $M \subset X \times U$, is the symmetry group of the system of differential equations (9), if its prolongation leaves the subvariety $\wp_{\Delta}^{(2)}$ invariant, i.e. for $\forall \mu \in \wp_{\Delta}^{(2)}$ it follows that $\mathbf{p r}^{(2)} g \mu \in \wp_{\Delta}^{(2)}$, where $\mathbf{p r}^{(2)} g$ is a second prolongation of the group $G$.

We will obtain the Lie algebra of infinitesimal generators $\mathrm{v}_{i}$ that meet the criteria of the infinitesimal invariance of the system (9) using known methods $[13,16]$ :

$$
\operatorname{pr}^{(2)} \mathrm{v}_{i}\left(\Delta_{v}(\mu)\right)=0, \quad v=1,2,3,
$$

where $\mu \in \wp_{\Delta}^{(2)}$.

The Lie algebra of infinitesimal generators of the system (9) symmetry groups is determined by 12 vector fields

$$
\begin{aligned}
& \mathbf{v}_{1}=\frac{\partial}{\partial x} ; \mathbf{v}_{2}=u_{0} \frac{\partial}{u_{0}} ; \quad \mathbf{v}_{3}=u_{1} \frac{\partial}{u_{1}} ; \mathbf{v}_{4}=u_{2} \frac{\partial}{u_{1}} ; \mathbf{v}_{5}=u_{1} \frac{\partial}{u_{2}} ; \mathbf{v}_{6}=u_{2} \frac{\partial}{u_{2}} ; \\
& \mathbf{v}_{7}=\sin \left(\sqrt{\frac{\lambda m_{0}}{p_{0}}} \cdot x\right) \frac{\partial}{u_{0}} ; \quad \mathbf{v}_{8}=\cos \left(\sqrt{\frac{\lambda m_{0}}{p_{0}}} \cdot x\right) \frac{\partial}{u_{0}} ; \quad \mathbf{v}_{9}=\sin \left(\sqrt{\frac{\lambda m_{s}}{p_{s}}} \cdot x\right) \frac{\partial}{u_{1}} \\
& \mathbf{v}_{10}=\cos \left(\sqrt{\frac{\lambda m_{s}}{p_{s}}} \cdot x\right) \frac{\partial}{u_{1}} ; \quad \mathbf{v}_{11}=\sin \left(\sqrt{\frac{\lambda m_{s}}{p_{s}}} \cdot x\right) \frac{\partial}{u_{2}} ; \quad \mathbf{v}_{12}=\cos \left(\sqrt{\frac{\lambda m_{s}}{p_{s}}} \cdot x\right) \frac{\partial}{u_{2}}
\end{aligned}
$$


One-parameter groups $G_{i}$ generated by the vector fields (11) $\mathrm{v}_{i}$ act as follows:

$$
\begin{aligned}
& G_{1}:\left(x+\varepsilon, u_{0}, u_{1}, u_{2}\right) ; \quad G_{2}:\left(x, e^{\varepsilon} u_{0}, u_{1}, u_{2}\right) ; \quad G_{3}:\left(x, u_{0}, e^{\varepsilon} u_{1}, u_{2}\right) ; \\
& G_{4}:\left(x, u_{0}, u_{1}+\varepsilon u_{2}, u_{2}\right) ; \quad G_{5}:\left(x, u_{0}, u_{1}, e^{\varepsilon} u_{2}\right) ; \quad G_{6}:\left(x, u_{0}, u_{1}, u_{2}+\varepsilon u_{1}\right) ; \\
& G_{7}:\left(x, u_{0}+\varepsilon \sin \left(\sqrt{\frac{\lambda m_{0}}{p_{0}}} \cdot x\right), u_{1}, u_{2}\right) ; \quad G_{8}:\left(x, u_{0}+\varepsilon \cos \left(\sqrt{\frac{\lambda m_{0}}{p_{0}}} \cdot x\right), u_{1}, u_{2}\right) ; \\
& G_{9}:\left(x, u_{0}, u_{1}+\varepsilon \sin \left(\sqrt{\frac{\lambda m_{s}}{p_{s}}} \cdot x\right), u_{2}\right) ; \quad G_{10}:\left(x, u_{0}, u_{1}+\varepsilon \cos \left(\sqrt{\frac{\lambda m_{s}}{p_{s}}} \cdot x\right), u_{2}\right) ; \\
& G_{11}:\left(x, u_{0}, u_{1}, u_{2}+\varepsilon \sin \left(\sqrt{\frac{\lambda m_{s}}{p_{s}}} \cdot x\right)\right) ; \quad G_{12}:\left(x, u_{0}, u_{1}, u_{2}+\varepsilon \cos \left(\sqrt{\frac{\lambda m_{s}}{p_{s}}} \cdot x\right)\right) .
\end{aligned}
$$

The group $G_{l}$ corresponds to invariance of solutions under translations along the $X$ axis. For the boundary value problems, this group is not a symmetry group. The groups $G_{2}, G_{3}, G_{5}$ illustrate the linearity of the operator $A$. The groups $G_{4}$ and $G_{6}$ represents the ability of permutation of the functions $u_{1}(x)$ and $u_{2}(x)$ in the system (9). The invariance of the solutions on the summation with the fundamental solutions of equations (9) is shown by the groups $G_{7}-G_{12}$.

Symmetry classification of the operator $\boldsymbol{A}$ eigenfunctions. Lie symmetry analysis allows us to formulate following result.

Theorem 2. Eigenfunctions of the operator $A$ are the basis functions of the irreducible representations of the group $S_{2}$.

Proof. It was shown above that besides the linearity of operator A functions $u_{1}(x), u_{2}(x)$ are permutable. Set of these permutations can be described as a finite group, isomorphic (by Cayley's theorem) to the group $S_{2}$.

Since invariance of equality $A U-\lambda U=0$ with respect to permutations has been shown in symmetry analysis of the system (9), then the invariance of problem (6) requires the invariance of the set $D(A)$ under group actions.

The group $S_{2}$ consists of two elements: the identity element $g_{I}$ and permutation $g_{1-2}$. In the space $H$, representation of the group $T\left(S_{2}\right)$ consists of two matrix operators $T\left(g_{I}\right)$ and $T\left(g_{1-2}\right)$, corresponding to elements of the group.

$$
T\left(g_{I}\right)=\left(\begin{array}{ccc}
1 & 0 & 0 \\
0 & 1 & 0 \\
0 & 0 & 1
\end{array}\right) ; \quad T\left(g_{1-2}\right)=\left(\begin{array}{ccc}
1 & 0 & 0 \\
0 & 0 & 1 \\
0 & 1 & 0
\end{array}\right) .
$$

Substitution of vector components $T\left(g_{I}\right) U$ and $T\left(g_{1-2}\right) U$ in equations (2) shows that the set $D(A)$ is invariant under the representation $T\left(S_{2}\right)$. Then for the problem (6) it follows that $A T\left(S_{2}\right) U_{k}-\lambda_{k} T\left(S_{2}\right) U_{k}=0$, where $U_{k}$ is an eigenelement of operator $A$ corresponding to its eigenvalue $\lambda_{k}$. 
As a result operator $A$ is commutative with the operators of the representation $T\left(S_{2}\right)$, and in this case, from the Wigner theorem [18] and Theorem 1 on the spectrum of the operator $A$, it follows that eigenfunctions of operator A are basis elements of the irreducible representations of the group $S_{2}$.

The characters $\chi^{(\alpha, \beta)}$ of the irreducible representations $\alpha$ and $\beta$ of the group $S_{2}$, and the characters $\chi^{(\tau)}$ of regular representation $\tau$ of the group $S_{2}$ in the space $H$ are shown in Table 1.

Table 1

Characters of representations of the group $S_{2}$

\begin{tabular}{|c|c|c|}
\hline Representation & $g_{\mathrm{I}}$ & $g_{1-2}$ \\
\hline$\alpha$ & 1 & 1 \\
\hline$\beta$ & 1 & -1 \\
\hline$\tau$ & 3 & 1 \\
\hline
\end{tabular}

Projectors on the subspaces of irreducible representations in space $H$ are given by

$$
\begin{aligned}
& P^{(\alpha)}=\frac{1}{2}\left(T\left(g_{I}\right)+T\left(g_{1-2}\right)\right) ; \\
& P^{(\beta)}=\frac{1}{2}\left(T\left(g_{I}\right)-T\left(g_{1-2}\right)\right) .
\end{aligned}
$$

For the eigenfunction $U_{k}$ that belongs to the subspace of $i$-th irreducible representation, take place following equations [19].

$$
P^{(i)} U_{k}=U_{k}
$$

It follows that the eigenfunctions of operator $A$ that belong to the subspace of irreducible representation $\alpha$ have the coordinates $\left\{u_{0}, u_{s}, u_{s}\right\}^{\mathrm{T}}$; the elements that belong to the subspace of irreducible representation $\beta$ have coordinates $\left\{0, u_{s},-u_{s}\right\}^{\mathrm{T}}$. It is also necessary to note that, by virtue of the theorem on the orthogonality of characters of irreducible representations [18], the spaces of irreducible representations are orthogonal. The subspaces of irreducible representations are found as follows:

$$
\begin{aligned}
& H=H^{(\alpha)} \oplus H^{(\beta)} ; \\
& H^{(\alpha)}=P^{(\alpha)} H ; \\
& H^{(\beta)}=P^{(\beta)} H .
\end{aligned}
$$

Solution of the spectral problem. Let us assume that

$$
\sqrt{\frac{\lambda m_{0}}{p_{0}}}=\Lambda, \sqrt{\frac{m_{s} p_{0}}{p_{s} m_{0}}}=a, \frac{p_{0}}{k}=b, \frac{p_{s}}{k}=c .
$$


Taking into account the boundary conditions (2) for coordinate $x=0$, the solutions are given by

$$
\begin{aligned}
& u_{0}(x)=C_{0} \cos (\Lambda \cdot x) \\
& u_{1}(x)=C_{1} \cos (a \Lambda \cdot x) \\
& u_{2}(x)=C_{2} \cos (a \Lambda \cdot x)
\end{aligned}
$$

For the subspace $H^{(\alpha)}$, we have $u_{0}(x)=C_{0} \cos (\Lambda x) ; \quad u_{1}(x)=u_{2}(x)=$ $=C_{1} \cos (a \Lambda x)$. Due to calculate eigenvalues $\lambda$ we obtain characteristic matrix $L^{(\alpha)}$ by substituting the functions (16) to the boundary conditions of (2) for the coordinate $x=l$ :

$$
L^{(\alpha)}=\left(\begin{array}{cc}
-\cos (\Lambda l) & -c a \Lambda \sin (a \Lambda l)+\cos (a \Lambda l) \\
-b \Lambda \sin (\Lambda l)+2 \cos (\Lambda l) & -2 \cos (a \Lambda l)
\end{array}\right) .
$$

The eigenvalues $\lambda$ corresponding to the elements of this subspace are the roots of the determinant of the matrix $L^{(\alpha)}$ :

$$
\begin{aligned}
\operatorname{det}\left(L^{(\alpha)}\right)= & b \Lambda \sin (\Lambda l)(\cos (a \Lambda l)-c a \Lambda \sin (a \Lambda l))+ \\
& +2 \cos (\Lambda l) c a \Lambda \sin (a \Lambda l)=0
\end{aligned}
$$

Let us assume that $C_{1}=1$. Eigenfunctions of the subspace $H^{(\alpha)}$ are given by

$$
\begin{gathered}
u_{0}(x)=-\frac{(\mathbf{c a} \Lambda \mathbf{s i n}(\mathbf{a} \Lambda \mathbf{l})-\cos (\mathbf{a} \Lambda \mathbf{l}))}{\cos (\Lambda l)} \cos (\Lambda x) ; \\
u_{1}(x)=u_{2}(x)=\cos (a \Lambda x) .
\end{gathered}
$$

Highlighting of the part of the coefficient for $u_{0}(x)$ in (19) was made for convenience of further statement.

For the subspace $H^{(\beta)}$ we have $u_{0}(x)=0, u_{1}(x)=-u_{2}(x)=C_{1} \cos (a \Lambda x)$. Similar to subspace $H^{(\alpha)}$ we obtain for subspace $H^{(\beta)}$ when $C_{1}=1$ :

$$
\begin{gathered}
L^{(\beta)}=(-c a \Lambda \sin (a \Lambda l)+\cos (a \Lambda l)) ; \\
\operatorname{det}\left(L^{(\beta)}\right)=-(\mathbf{c a} \Lambda \sin (\mathbf{a} \Lambda \mathbf{l})-\cos (\mathbf{a} \Lambda \mathbf{l}))=0 ; \\
u_{0}(x)=0 ; \\
u_{1}(x)=-u_{2}(x)=\cos (a \Lambda l) .
\end{gathered}
$$

Now let us will consider the solution of the spectral problem (6) in the initial space $H$. By substituting the functions (16) into the boundary conditions of (2) for the coordinate $x=l$, we write characteristic matrix for the calculation of the eigenvalues $\lambda$ : 


$$
L=\left(\begin{array}{ccc}
-\cos (\Lambda l) & -c a \Lambda \sin (a \Lambda l)+\cos (a \Lambda l) & 0 \\
-\cos (\Lambda l) & 0 & -c a \Lambda \sin (a \Lambda l)+\cos (a \Lambda l) \\
-b \Lambda \sin (\Lambda l)+2 \cos (\Lambda l) & -\cos (a \Lambda l) & -\cos (a \Lambda l)
\end{array}\right)
$$

Eigenvalues $\lambda$ are the roots of the matrix $L$ determinant:

$$
\begin{aligned}
\operatorname{det}(L)=(\mathbf{c a} \Lambda \sin (\mathbf{a} \Lambda \mathbf{l}) & -\cos (\mathbf{a} \Lambda \mathbf{l}))(b \Lambda \sin (\Lambda l)(\cos (a \Lambda l)-c a \Lambda \sin (a \Lambda l))+ \\
& +2 \cos (\Lambda l) c a \Lambda \sin (a \Lambda l))=0 .
\end{aligned}
$$

Let us assume that $C_{2}=1$, then the eigenfunctions (16) are written as follows:

$$
\begin{gathered}
u_{0}(x)=-\frac{(\mathbf{c} \mathbf{\Lambda} \mathbf{s i n}(\mathbf{a} \Lambda \mathbf{l})-\cos (\mathbf{a} \Lambda \mathbf{l}))}{\cos (\Lambda l)} \cos (\Lambda x) ; \\
u_{1}(x)= \\
=\frac{(b \Lambda \sin (\Lambda l)-\cos (\Lambda l))(\mathbf{c a} \Lambda \mathbf{s i n}(\mathbf{a} \Lambda \mathbf{l})-\cos (\mathbf{a} \Lambda \mathbf{l}))-\cos (\Lambda l)(c a \Lambda \sin (a \Lambda l))}{\cos (\Lambda l) \cos (a \Lambda l)} \times \\
\times \cos (a \Lambda x) ; \\
u_{2}(x)=\cos (a \Lambda x) .
\end{gathered}
$$

The expression (22) for the determinant of matrix $L$ consists of two multipliers, the first of them is highlighted in bold and equal to $\operatorname{det}\left(L^{(\beta)}\right)$, up to sign. If this multiplier is equal to zero, then found eigenvalues will coincide with the eigenvalues corresponding to the eigenvalues of the subspace $H^{(\beta)}$. In this case, in (23) the function $u_{0}(x)$ will be identically zero, and $u_{1}(x)$ will be equal to $-u_{2}(x)$.

The second multiplier in (22) coincides with $\operatorname{det}\left(L^{(\alpha)}\right)$ and the eigenfunctions corresponding to zeroes of this multiplier will belong to $H^{(\alpha)}$. The coefficient of $\cos (a \Lambda x)$ in (23) becomes equal to one.

In addition to the decomposition of the initial space to subspaces of smaller dimension, we can divide the whole spectrum $\sigma(A)$ of the operator $A$ to corresponding subspectra

$$
\sigma(A)=\sigma^{(\alpha)}(A) \cup \sigma^{(\beta)}(A)
$$

defining equations of which (18), (20) have simpler form than initial one (22).

In summary the group-theoretic approach shown above has allowed us to simplify the solution of the spectral problem. 
Numerical example. Let us assume that $a=1,3, b=2,5, c=0,7, l=1$. The diagrams $\operatorname{det}\left(L_{(\alpha)}\right)$ and $\operatorname{det}\left(L_{(\beta)}\right)$ are shown at the figures $2 a$ and $2 b$, respectively. Diagrams for $\operatorname{det}\left(L_{(\alpha)}\right), \operatorname{det}\left(L_{(\beta)}\right)$ and $\operatorname{det}(L)$ are shown at figure $2 c$.

We will give the values $\Lambda$ corresponding to the first 4 eigenvalues of the operator A (see Table 2) and diagrams of eigenfunctions corresponding to them (Fig. $3 a-d)$. Here eigenfunctions were normalized by their maximum value in $[0, l]$.

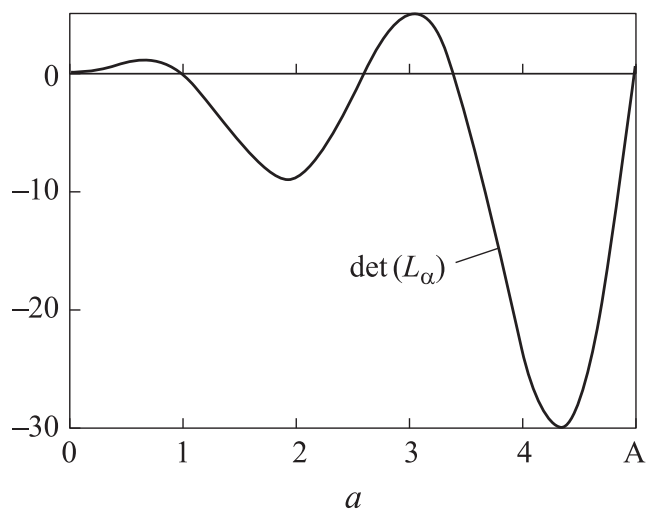

Fig. 2a. Diagram of $\operatorname{det}\left(L_{(\alpha)}\right)$

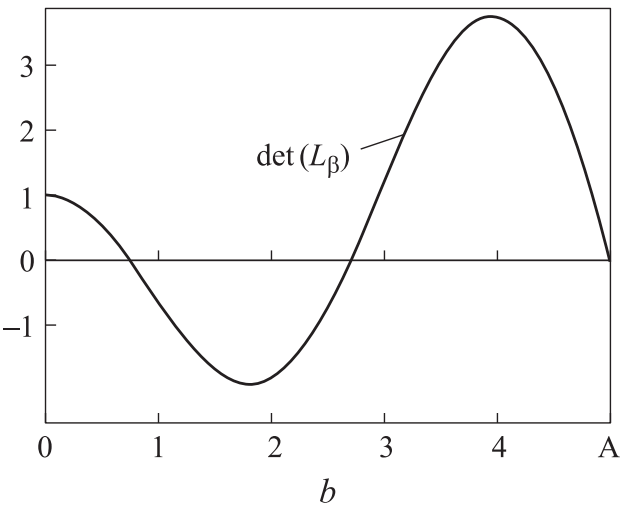

Fig. 2b. Diagram of $\operatorname{det}\left(L_{(\beta)}\right)$

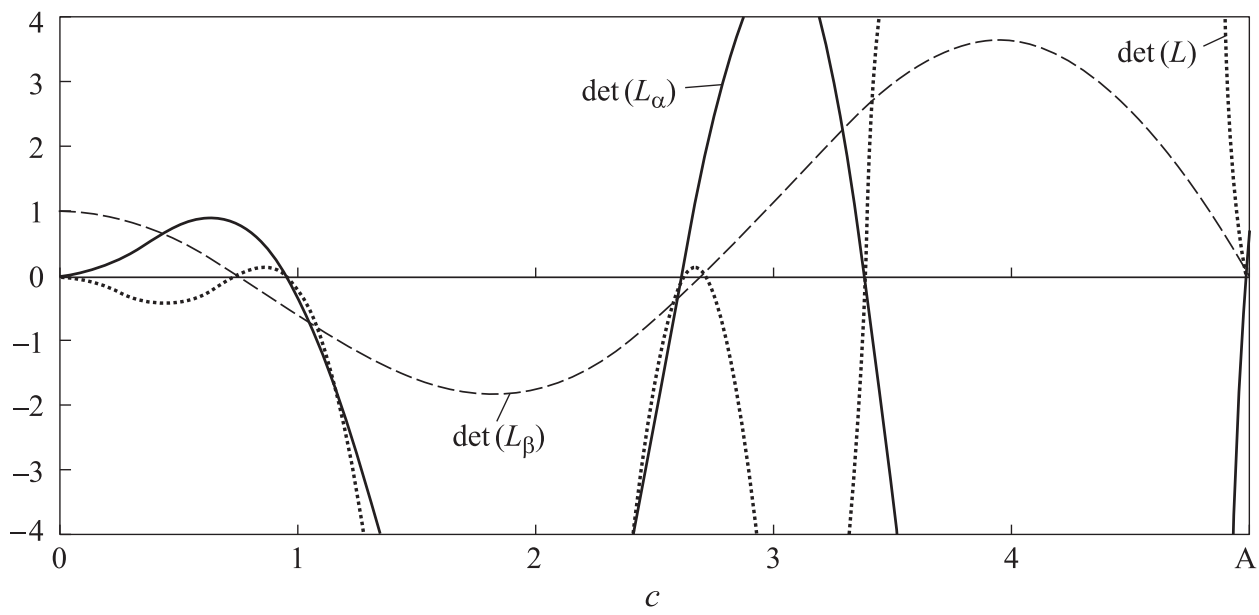

Fig. 2c. Combined diagram of $\operatorname{det}\left(L_{(\alpha)}\right), \operatorname{det}\left(L_{(\beta)}\right), \operatorname{det}(L)$

Table 2

Eigenvalues of the operator $A$

\begin{tabular}{|c|c|c|}
\hline No. & $\Lambda$ & Subspectrum \\
\hline 1 & 0,75 & $\sigma^{(\beta)}(A)$ \\
\hline 2 & 0,95 & $\sigma^{(\alpha)}(A)$ \\
\hline 3 & 2,62 & $\sigma^{(\alpha)}(A)$ \\
\hline 4 & 2,71 & $\sigma^{(\beta)}(A)$ \\
\hline
\end{tabular}



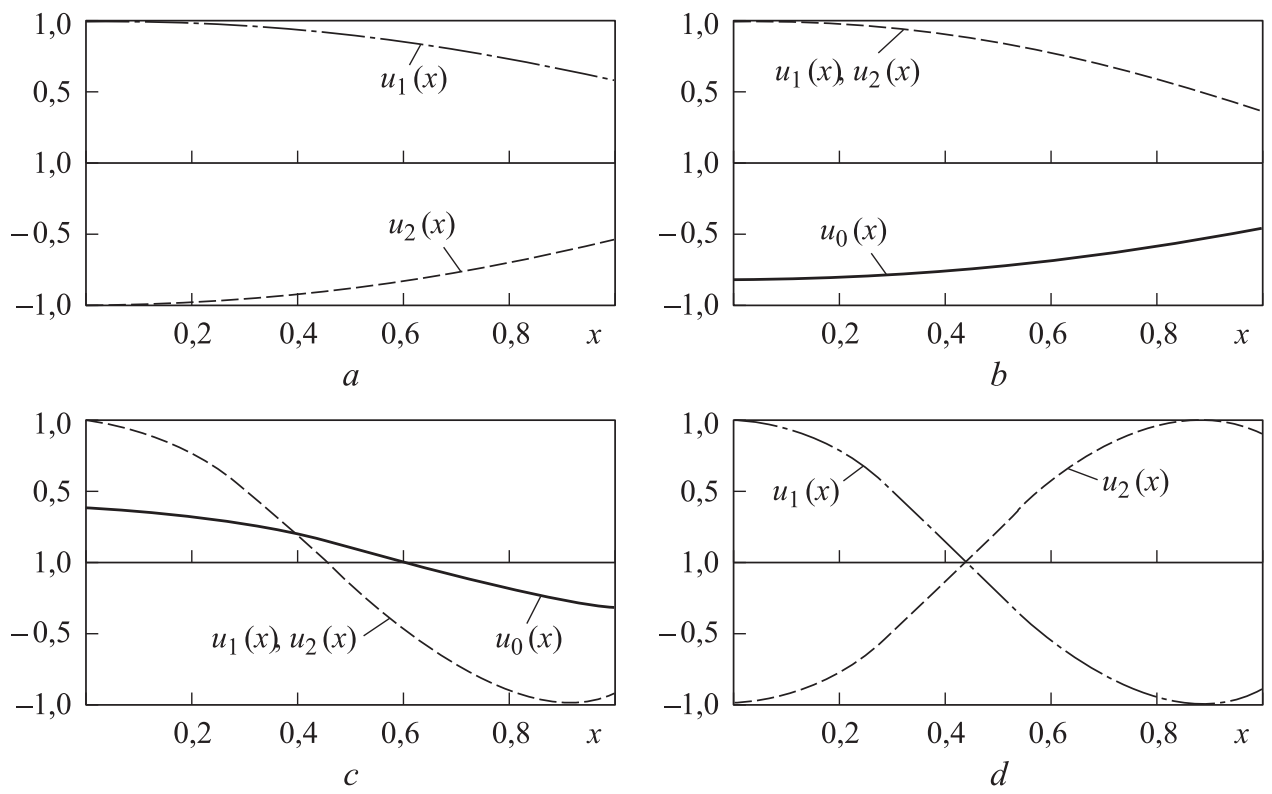

Fig. 3. $(a-\Lambda=0,75 ; \sigma-\Lambda=0,95 ; c-\Lambda=2,62 ; d-\Lambda=2,71)$

Summary of the results obtained above is written in the form of the following theorem.

Theorem 3. Spectrum of the operator A consists of two subspectra:

$$
\sigma(A)=\sigma^{(\alpha)}(A) \cup \sigma^{(\beta)}(A) .
$$

Eigenfunctions of the operator belong to the subspaces $H^{(\alpha)}$ and $H^{(\beta)}$ respectively, so that $H=H^{(\alpha)} \oplus H^{(\beta)}, H^{(\alpha)}=P^{(\alpha)} H, H^{(\beta)}=P^{(\beta)} H$ where $P^{(\alpha)}, P^{(\beta)}$ are orthogonal projectors onto subspaces of irreducible representations of the symmetric group $S_{2}$.

Solution of the Cauchy problem. If the initial conditions and the right side of the Cauchy problem considered above, transforms under irreducible representations of group $S_{2}$ (e. g. belongs to spaces of these representation) results of symmetry classification of the corresponding spectral problem eigenvalues are useful in following solution and analysis of the problem (5).

We will consider a weak solution of the problem (5). In the metric of space $\tilde{H}=H \oplus\{1\}$, operator $A$ eigenfunctions decomposition of the solution $U(t)$ is given by:

$$
U(t)=\sum_{k=0}^{\infty} \varphi_{k}(t) U_{k}, \varphi_{k}(t)=\left(U(t), U_{k}\right)
$$

where the coefficients $\varphi_{k}(t)$ are solutions of the Cauchy problems

$$
\begin{gathered}
\frac{d^{2} \varphi_{k}(t)}{d t^{2}}+\lambda_{k} \varphi_{k}(t)=f_{k}(t), \quad k=0 \ldots \infty \\
\varphi_{k}(0)=\left(\Psi^{0}, U_{k}\right), \quad \dot{\varphi}_{k}(0)=\left(\Psi^{1}, U_{k}\right), \quad f_{k}(t)=\left(F(t), U_{k}\right) .
\end{gathered}
$$


Here $U_{0}=\{1\}, \lambda_{0}=0$ is the solution of the system (9), orthogonally complementing space $H$. It is easy to find that $U_{0}$ transforms according to the irreducible representation $\alpha$ of the group $S_{2}$.

Taking into account (25) and (26), weak solution of problem (5) is written in the following form [20]

$$
\begin{aligned}
& U(t)=\sum_{k=0}^{\infty}\left(\left(\Psi^{0}, U_{k}\right) \cos \left(\sqrt{\lambda_{k}} t\right)+\frac{\left(\Psi^{1}, U_{k}\right)}{\sqrt{\lambda_{k}}} \sin \left(\sqrt{\lambda_{k}} t\right)+\right. \\
&\left.+\frac{1}{\sqrt{\lambda_{k}}} \int_{0}^{t} \sin \left(\sqrt{\lambda_{k}}(t-s)\right)\left(F(t), U_{k}\right) d s\right) U_{k}+ \\
&+\left(\int_{0}^{t} \int_{0}^{s}\left(F(s), U_{0}\right) d s d s+\left(\Psi^{1}, U_{0}\right) t+\left(\Psi^{0}, U_{0}\right)\right) U_{0} .
\end{aligned}
$$

We will decompose $F(t), \Psi^{0}, \Psi^{1}$ on the basis of the group $S_{2}$ irreducible representations

$$
\begin{gathered}
F(t)=\sum_{v=\alpha, \beta} P^{(v)} F(t)=\sum_{v=\alpha, \beta} F^{(v)}(t) ; \\
\Psi^{0}=\sum_{v=\alpha, \beta} P^{(v)} \Psi^{0}=\sum_{v=\alpha, \beta} \Psi^{0(v)} ; \\
\Psi^{1}=\sum_{v=\alpha, \beta} P^{(v)} \Psi^{1}=\sum_{v=\alpha, \beta} \Psi^{(1(v)} .
\end{gathered}
$$

Since the subspaces $H^{(\alpha)}$ and $H^{(\beta)}$ are orthogonal, the scalar products where non-zero projections of vectors $F(t), \Psi^{0}, \Psi^{1}$ on one side and the eigenfunction $U_{k}$ on the other side, belong to different subspaces, will be zeroed out. If in (28), projections of the vectors $F(t), \Psi^{0}, \Psi^{1}$ on any of the subspaces will be simultaneously equal to zero then in the weak solution of (27), we can neglect the subspectra and corresponding eigenfunctions that belong to subspaces where projections of vectors $F(t), \Psi^{0}, \Psi^{1}$ are both equal to zero.

Conclusion. The group-theoretic approach considered in this work is useful in solution and analysis of the more complex spectral and evolutionary problems generated by vibrations of symmetrical rod and beams systems, presented, for example, in [21].

Symmetry classification of eigenfunctions of the boundary value problem operator allows to simplify eigenvalues calculation process via solving the problem in subspaces of lower dimension than the initial space. Hence, the spectrum is divided into subspectra corresponding to the elements of these subspaces. As a benefit asymptotic behavior for each of the subspectra can be obtained.

In the weak solution of the evolutionary problem, orthogonality of the obtained subspaces admits not to use eigenfunctions that belong to those subspaces where 
projections of the initial conditions and the right-hand side of the equation are equal to zero at the same time. In case of limitations on the number of eigenfunctions used, this approach increases convergence of the solution to the exact one.

\section{REFERENCES}

[1] Miller W. Symmetry groups and their applications. New York, Academic Press, 1972. $436 \mathrm{p}$

[2] Zlokovic G. Group theory and G-vector spaces in the structural analysis. Chichester, UK, Ellis Horwood, 1989. 283 p.

[3] Zingoni A. Group-theoretic insights on the vibration of symmetric structures in engineering. Phil. Trans. R. Soc. A, 2014, no. 372. DOI: 10.1098/rsta.2012.0037

Available at: http://rsta.royalsocietypublishing.org/content/372/2008/20120037

[4] Healey T., Treacy J. Exact block diagonalization of large eigenvalue problems for structures with symmetry. Int. J. Numer. Meth. Eng., 1991, vol. 31, no. 2, pp. 265-285.

[5] Herzberg G. Infrared and Raman spectra of polyatomic molecules. New York, Van Nostrand, $1945.632 \mathrm{p}$.

[6] Kaveh A., Nikbakht M. Decomposition of symmetric mass-spring vibrating systems using groups, graphs and linear algebra. Numer. Meth. Biomed. Eng., 2007, vol. 23, no. 7, pp. 639-664.

[7] Kaveh A., Nikbakht M. Improved group-theoretical method for eigenvalue problems of special symmetric structures, using graph theory. Adv. Eng. Softw., 2010, vol. 41, no. 1, pp. 22-31.

[8] Mohan S., Pratap R. A Group theoretic approach to the linear free vibration analysis of shells with dihedral symmetry. J. Sound Vib., 2002, vol. 252, no. 2, pp. 317-341.

[9] Mohan S., Pratap R. A natural classification of vibration modes of polygonal ducts based on group theoretic analysis. J. Sound Vib., 2004, vol. 269, no. 3-5, pp. 745-764.

[10] Zingoni A., Pavlovic M., Lloyd-Smith D., Zlokovic G. Group-theory considerations of finite-difference plate eigenvalue problems. In: Developments in computational engineering mechanics. 1993. Pp. 243-256.

[11] Bunker P., Jensen P. Molecular symmetry and spectroscopy. Ottawa, NRC research press, 1998. 747 p.

[12] Zingoni A. Group-theoretic exploitations of symmetry in computational solid and structural mechanics. Numer. Meth. Eng., 2009, no. 79, pp. 253-289.

[13] Olver P. Applications of Lie groups to differential equations. New York, Springer-Verlag, 1986. 497 p.

[14] Barut A., Raczka R. Theory of group representations and applications. Singapore, World Scientific, 1986. 717 p.

[15] Asherova R.M., Smirnov Y.F. Projection operators for classical groups. UMN, 1969, vol. 24, no. 3(147), pp. 227-228 (in Russ.).

[16] Ovsiannikov L.V., Ames W. Group analysis of differential equations. New York, Academic Press, 1982. 416 p.

[17] Pavlov A.M., Temnov A.N. System of rods longitudinal vibrations. Vestn. Mosk. Gos. Tekh. Univ. im. N.E. Baumana, Estestv. Nauki [Herald of the Bauman Moscow State Tech. Univ., Nat. Sci.], 2014, no. 6, pp. 53-66 (in Russ.). 
[18] Wigner E. Group theory and its application to the quantum mechanics of atomic spectra. New York, Academic Press, 1959. 372 p.

[19] Hamermesh M. Group theory and its application to physical problems. New York, Dover, 1989. 509 p.

[20] Mikhlin S.G. Linear equations of mathematical physics. New York, Holt, Rinehart and Winston, $1967.318 \mathrm{p}$.

[21] Zimin V.N., Krylov A.V., Meshkovskii V.E., Sdobnikov A.N., Fayzullin F.R., Churilin S.A. Features of the calculation deployment large transformable structures of different configurations. Nauka i obrazovanie: nauchnoe izdanie MGTU im. N.E. Baumana [Science and Education: Scientific Publication of BMSTU], 2014, no. 10, pp. 179-191 (in Russ.).

DOI: 10.7463/1014.0728802 Available at: http://technomag.edu.ru/jour/article/view/702

Pavlov A.M. - post-graduate student of Spacecraft and Launch Vehicles Department, Bauman Moscow State Technical University (2-ya Baumanskaya ul. 5, str. 1, Moscow, 105005 Russian Federation).

Temnov A.N. - Cand. Sc. (Phys.-Math.), Assoc. Professor of Spacecraft and Launch Vehicles Department, Bauman Moscow State Technical University (2-ya Baumanskaya ul. 5, str. 1, Moscow, 105005 Russian Federation).

\section{Please cite this article in English as:}

Pavlov A.M., Temnov A.N. Symmetry Exploitation in the Natural Vibrations of Rod Systems. Vestn. Mosk. Gos. Tekh. Univ. im. N.E. Baumana, Mashinostr. [Herald of the Bauman Moscow State Tech. Univ., Mech. Eng.], 2017, no. 4, pp. 28-41.

DOI: 10.18698/0236-3941-2017-4-28-41 